\title{
Antenatally diagnosed congenital anomalies-trend in tertiary care hospital
}

\author{
Kamal Kishore ${ }^{1}$, Madhusudan Dey ${ }^{2}$, Ashisha Gaba ${ }^{3}$, Shijith K. P. ${ }^{4}$, Reema Kumar Bhatt ${ }^{5 *}$
}

\author{
${ }^{1}$ Department of Paediatric Surgery, Command Hospital, Lucknow, Uttar Pradesh, India \\ ${ }^{2}$ Department of Fetal medicine, Base hospital, Delhi Cantt., New Delhi, India \\ ${ }^{3}$ Department of Obstetrics and Gynecology, ${ }^{4}$ Department of Radiodiagnosis, ${ }^{5}$ Department of Fetal Maternal Medicine \\ Subunit, Army Hospital Research and Referral, Delhi, India
}

Received: 30 January 2021

Accepted: 15 February 2021

\section{*Correspondence:}

Dr. Reema Kumar Bhatt,

E-mail: reemakumarbhatt@gmail.com

Copyright: ( $)$ the author(s), publisher and licensee Medip Academy. This is an open-access article distributed under the terms of the Creative Commons Attribution Non-Commercial License, which permits unrestricted non-commercial use, distribution, and reproduction in any medium, provided the original work is properly cited.

\begin{abstract}
Background: Antenatally diagnosed, congenital abnormalities of the fetus are frequently offered antenatal surgical consultation in all tertiary care hospitals to adequately counsel prospective parents. We aimed to assess the current trends in antenatally diagnosed congenital malformations of fetus in patients who had presented to our hospital for reassessment or surgical consultation and these patients were counselled and prognosticated after confirmation of anomaly at our centre accordingly.

Methods: This was a retrospective study of all the cases diagnosed antenatally with fetal abnormalities. All cases were referred to either pediatric surgery outpatient department or fetal medicine subunit of a tertiary care centre from January 2017-December 2018. The complete records of these pregnant women were perused, presence and type of anomaly confirmed at our centre and trends about types of anomalies analysed.

Results: A total of 209 women were analyzed for antenatal anomalies, out of these patients medical termination of pregnancy was offered to $27(12.9 \%)$ patients, in which 38 anomalies were detected and in 182 (87\%) patients pregnancy was continued to delivery. A total of 223 anomalies were detected in this group with some patients having fetuses with more than one anomaly. Gastrointestinal abnormalities were present in 94 (51.6\%) patients of those who delivered and accounted for the most common anomaly followed by CNS abnormality which accounted for 39 $(21.4 \%)$ of total abnormalities and genitourinary abnormalities contributed to $28(13.3 \%)$ of abnormalities. A total of $85.2 \%$ of patients were referred after 20 weeks.

Conclusions: The significant number of patients with major congenital anomalies are still diagnosed at a date later than permissible time for termination of gestation (20 weeks). There were cases where anomaly scan was done at a later gestation and then there were others where inadequate counselling made patient to present late for prognostication. However, there are certain anomalies that are picked up late and are likely to be missed in the routine anomaly scan. Therefore, antenatal detection and early referral helps in offering early counselling and better outcome for pregnancy.
\end{abstract}

Keywords: Antenatally diagnosis, Major anomalies, Pediatric consultation, Tertiary care hospitals

\section{INTRODUCTION}

In the current era of better awareness, better machines, improvised techniques and wider availability of ultrasound, congenital anomalies still remain a challenge. ${ }^{1,2}$ Antenatal anomalies are now mostly being diagnosed in the first trimester in expert hands. The early detection of serious malformations provides us with a window for termination of pregnancy after counselling of expectant parents. ${ }^{2}$ In spite of this high surveillance and awareness there are abnormalities that escape detection due to diagnosis in the late second or third trimester, 
especially some of those involving gastrointestinal and genitourinary system and some of central nervous system as well. ${ }^{3}$ There is an increased need today to plan the feasible postnatal management of these malformations to increase the preparedness of the pediatric surgery team and also of parents, especially in late detection beyond 20 weeks of gestation where there is no option but continuation of pregnancy.

In India, incidence of pregnancy with congenital anomalies and perinatal mortality has varied greatly as compared to other developed countries. These anomalies accounts for $10-15 \%$ of perinatal deaths and $13-16 \%$ of neonatal deaths. ${ }^{4,5}$ There are also very few reports on the anomalies detected antenatally being offered pediatric surgery counselling.

A retrospective study was conducted in the pediatric surgery outpatient department and fetal medicine subunit of a tertiary care hospital in New Delhi to assess the antenatal diagnosis of congenital anomalies in the patients and offer them the counselling. Herein, we analysed different anomalies diagnosed and referred at the different gestational age, expectant parents were counselled regarding outcome depending upon the type of anomaly and whether there was scope of offering postnatal therapy and termination or continuation of pregnancy were advised depending upon severity and predicted outcome of pregnancy and also timing of the diagnosis.

\section{METHODS}

This was an observational study with retrospective analysis carried out between January 2017 to December 2018 on pregnant women $(n=209)$ referred to the pediatric surgery outpatient department and fetal medicine subunit of a tertiary care centre.

\section{Inclusion criteria}

As a part of the study, expectant mothers referred to the centre, underwent repeat ultrasonography (USG) scans, irrespective of gestational age. Fetal magnetic resonance imaging (MRI) was resorted to in certain cases for detailed evaluation of any structural abnormality if deemed necessary. The fetuses diagnosed with cardiac anomalies were referred to pediatric cardiologist and were also not included in the study.

\section{Exclusion criteria}

Further, patients without confirmatory ultrasound scans or those with incomplete antenatal records were excluded from the study.

\section{Ethical approval}

This study was performed under the norms of ethical approval from the institutional ethical committee.
The USG at our centre was performed with a lowfrequency $(2-5 \mathrm{MHz})$ convex probe. In addition to searching for anomalies, a detailed assessment of all body systems was done. In the case, where there was a doubtful finding such as non-visualized or distended stomach/urinary bladder, repeat USG was performed on different occasions. Patients underwent other investigations also such as fetal echocardiography, an infection screen like TORCH testing, biochemical screening for aneuploidy and genetic counselling as per ultra-sonography findings at the fetal medicine subunit of our hospital. Details related to their previous scans, gestational age of the fetus or any advice given for immediate and long term prognosis were collected from the parents.

The anomalies that were accounted for fetus were gastrointestinal (GIT), genitourinary (GUT), central nervous system (CNS), thoracic and others malformations. To examine the effect of anomalies, at different period of gestation, the patients were divided in (a) <20 weeks gestation, (b) > 20 week gestation groups. The chance of occurrence of anomalies at different gestational age allows the patient to undergo the termination before 20 weeks if diagnosed and to continue the pregnancy if diagnosed later. Moreover, the patients were contacted when hospital information was incomplete or for any follow ups for affected cases. Information gathered on medical condition diagnosed and interventions given were recorded on the regular basis.

The following were considered to have anomalies with poor prognostic outcome: congenital diaphragmatic hernia $(\mathrm{CDH})$ with liver in the thorax, lung to head ratio (LHR) <1.4, bilateral hydroureteronephrosis with distended bladder and oligohydramnios, bilateral multicystic kidney disease with oligohydramnios, anencephaly, gross hydrocephalus, neural tube defects, congenital cystic adenomatoid malformation of lung with associated congenital pulmonary airway malformation volume ratio (CVR) >1.4 and those with multiple anomalies.

\section{Statistical analysis}

Data has been analysed using statistical package for social sciences. Data has been represented as frequency (numbers) and proportions (percentages).

\section{RESULTS}

Over the period of two years, 209 pregnant women were screened with detection of 38 anomalies in MTP group and 221 anomalies in fetuses which continued to delivery. The prenatal finding of 209 cases were analysed for the occurrence of anomalies within the fetal GIT, GUT, CNS, thorax and other miscellaneous anomalies respectively. Among the total cases $31(14.8 \%)$ patients presented before 20 weeks and $178(85.2 \%)$ patients presented after 20 weeks of gestation (Figure 1). 


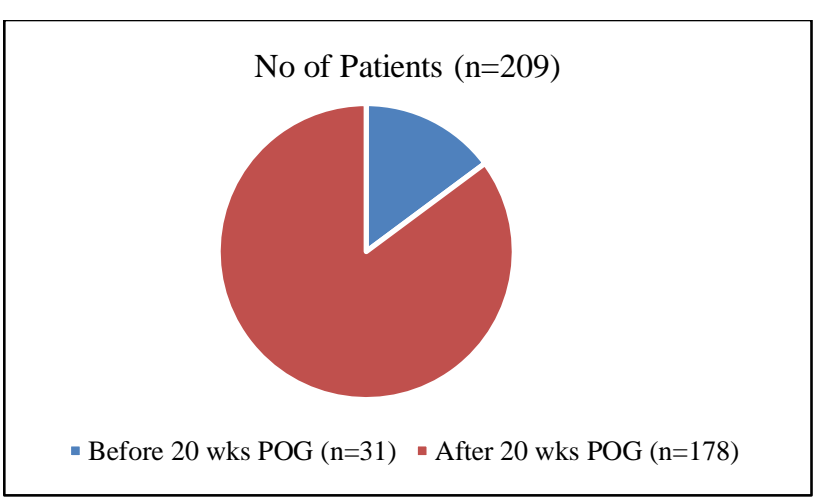

Figure 1: Distribution of patients as per POG.

Of the total cases enrolled the GIT anomalies were the most frequent anomalies amounting to 99 (47.4\%) cases in the complete cohort of 209 patients and $94(51.6 \%)$ cases in 182 completed pregnancies. GIT anomalies were also one of the most common cause of neonatal death which claimed $21(63.6 \%)$ lives out of 33 deaths in neonates and infants. This was followed by CNS anomalies which amounted to $50(23.9 \%)$ cases in complete cohort and $39(21.4 \%)$ cases in the pregnancies culminating in delivery. Also CNS anomalies were one of the most common anomalies in the MTP group with 11 $(40.7 \%)$ cases in 27 patients. GUT malformations were seen in $34(16.3 \%)$ of subjects with $28(15.4 \%)$ occurring in the delivered group. Anomalies involving the fetal thorax were least common, occurring in $9(4.3 \%)$ of total subjects and $8(4.4 \%)$ of delivered neonates. A significant number of patients had other anomalies e.g. chromosomal anomalies, malformation of limbs, large arterio-venous or lymphatic malformations and conjoint twins which were seen in $69(33 \%)$ of cases and $54(29.7 \%)$ of the delivered babies (Table 1).

Table 1: System wise distribution of anomalies.

\begin{tabular}{|lllllll|}
\hline & GIT & GUT & CNS & Chest & Others & Total anomalies \\
\hline MTP $(\mathbf{n}=\mathbf{2 7})$ & $5(18.5 \%)$ & $6(22.2 \%)$ & $11(40.7 \%)$ & $1(3.7 \%)$ & $15(55.5 \%)$ & $38(1.4$ per patient $)$ \\
\hline Delivered $(\mathbf{n = 1 8 2})$ & $94(51.6 \%)$ & $28(15.4 \%)$ & $39(21.4 \%)$ & $8(4.4 \%)$ & $54(29.7 \%)$ & $223(1.2$ per patient $)$ \\
\hline Total $(\mathbf{n = 2 0 9})$ & $99(47.4 \%)$ & $34(16.3 \%)$ & $50(23.9 \%)$ & $9(4.3 \%)$ & $69(33 \%)$ & $261(1.2$ per patient $)$ \\
\hline
\end{tabular}

\section{DISCUSSION}

With advances in the fetal ultrasonography and imaging along with raised awareness of both the medical practitioners and expectant parents most congenital malformations can be diagnosed antenatally in utero at early gestational period. ${ }^{6}$ This not only simplifies the management of pregnancy but also minimizes adverse outcomes for the fetus and informed decision making by the parents. The presence and prevalence of various abnormalities in GUT, GIT, Thoracic cavity and CNS and other systems of the fetus at different gestational ages was assessed in this study. Therefore, this study provides an insight into present day trends in clinical detection of different anomalies associated with the fetus along with areas which require introspection. The data for the occurrence of different anomalies indicates that with the improved ultrasound resolution and ever improving expertise of radiologists and fetal medicine experts many of the lesions can be picked up early in antenatal period. Early detection of anomalies can help in taking informed decision regarding termination or continuation of pregnancy by expectant parents, if the pregnancy is beyond the permissible POG for termination or so desired by the parents, and timely referral for those requiring emergent and life-saving interventions to appropriate centres, also anomalies that are detected timely improves the outcome of the fetus.

The severe and disabling malformations that are picked early were terminated after counselling of parents, but only 31 of 209 anomalies were referred before 20 weeks POG and 27 of these were terminated. Those that were diagnosed later were referred to pediatric surgical OPD. The overall perinatal anomalies in the study $(n=209)$, followed GIT $>$ CNS $>$ GUT $>$ thoracic trend in the percentage of occurrence. It indicated that the detection of GIT abnormalities is challenging and may not always show up ultrasonographically in early trimesters. ${ }^{3}$ The findings of the present study showed the significant number of GIT abnormalities were detected late e.g. $>20$ weeks gestations. Similar study was reported by Menon et al. ${ }^{3}$ In their study $38 \%$ of patients reported before 20 weeks of gestation as compared to $14.8 \%$ patients in our study, also major anomalies of GUT were seen in $46 \%$ of patients and were the predominant anomalies as compared to GIT anomalies seen in our study, this indicates regional differences in both patterns of anomaly detection and presentation dependent upon the population base. Further, reliable data on long term outcomes and quality of life in patients with antenatally detected congenital anomalies are limited. The current study showed that detection of some anomalies especially corresponding to GIT is technically challenging in early neonatal period and which may also be complicated by natural history of the disease rather than due to technical limitations only. ${ }^{8}$ Another aspect to late presentation could be failure to understand the implications of antenatally detected findings as well as consequences of late presentation by the couples. Some of the anomalies may have been missed due to lack of optimal conditions for detecting them. 
The $12.9 \%$ cases before 20 weeks $(n=27)$ undertook medical termination of pregnancy. The Medical Termination of Pregnancy Act of 1971 of India allows termination of pregnancy only before 20 weeks gestational age so $89.9 \%$ of cases had to continue with their pregnancy. The $18.1 \%$ of the neonates showed adverse outcomes in all the pregnancies continued to term and of those, anomalies involving the GIT e.g. intestinal atresias, large omphalocele etc. resulted in $11.5 \%$ of the total demises, followed by anomalies of CNS whereas in MTP group, CNS anomalies were predominant reason, $40.7 \%$, for termination. Timely counselling and intervention lead to survival in $71.3 \%$ of neonates in this cohort. This study also indicated that gestation life span contributed significant influence on the outcome of the treatment. The early detection of the radiological evidences for GIT and CNS malformations will be beneficial to offer treatment that will results in most satisfactory outcomes. The most important factor is the general health status of the fetus and severity of the anomalies in the fetus. Over the past few years, the trends in early diagnosis of congenital malfunctions showed significant improvements in the survival rates and also the fact that prognostication of disease where the counselling about progression or regression of abnormality like a fetus with $\mathrm{CDH}$, if there is no liver herniation and the LHR is more than 1.4, delivery at term in a tertiary care hospital with neonatal intensive care facilities has a good prognosis. ${ }^{3}$

On the other hand, association with aneuploidy and other associated findings, especially cardiac and marked pulmonary hypoplasia prognosticates the fetus adversely. Similarly, in GUT abnormality bilateral gross hydroureteronephrosis with oligohydramnios with suspicion of posterior urethral valves detected in second trimester has a poor prognosis, while unilateral or bilateral hydronephrosis will be expected to have good prognosis unless associated with some other abnormality.

\section{CONCLUSION}

According to the study performed at our hospital, the significant number of patients with fetal anomalies were diagnosed at a late POG (after 20 weeks). Also, there are regional variations in trends of fetal anomaly detection and presentation to a tertiary centres. With improved awareness, better USG machines and better training, it may be possible to offer the diagnosis at early gestational ages that could entail early referral and safe termination. However, there are certain anomalies that are picked up late and are likely to be missed in the routine anomaly scan. Therefore, antenatal detection and early referral helps in offering early intervention and does help to improve the outcome of pregnancy.

Funding: No funding sources

Conflict of interest: None declared

Ethical approval: The study was approved by the Institutional Ethics Committee

\section{REFERENCES}

1. Redkar R, Davenport M, Howard ER. Antenatal diagnosis of congenital anomalies of the biliary tract. J Pediatr Surg. 1998;33(5):700-4.

2. Sallout BI, Al Hoshan MS, Attyyaa RA, Al Suleimat AA. Antenatal diagnosis, prevalence and outcome of major congenital anomalies in Saudi Arabia: a hospital-based study. Ann Saudi Med. 2008;28(4):272-6.

3. Menon P, Binu V, Rao KLN, Suri V. Trends in referral pattern of antenatally diagnosed, surgical abnormalities in a tertiary care center in North India. J Indian Assoc Pediatr Surg. 2018;23(4):198-202.

4. Agarwal SS, Singh U, Singh PS, Singh SS, Das V, Sharma A, et al. Prevalence and spectrum of congenital malformations in a prospective study at a teaching hospital. Indian J Med Res. 1991;94:413-9.

5. Cleone R, World Health Organization. Maternal Health and Safe Motherhood Programme. (1992. Antenatal care and maternal health: how effective is it? A review of the evidence. World Health Organization. Available from: https://apps.who.int/iris/handle/10665/59954. Accessed on 3 September 2020.

6. Grandjean H, Larroque D, Levi S. The performance of routine ultrasonographic screening of pregnancies in the Eurofetus Study. Am J Obstet Gynecol. 1999;181(2):446-54.

7. Saini N, Chhabra S, Chhabra S, Garg L, Garg N. Pattern of neonatal morbidity and mortality: A prospective study in a District Hospital in Urban India. J Clin Neonatol. 2016;5(3):183-8.

8. Puri P, Fujimoto T. New observations on the pathogenesis of multiple intestinal atresias. J Pediatr Surg. 1988;23(3):221-5.

Cite this article as: Kishore K, Dey M, Gaba A, Shijith KP, Bhatt RK. Antenatally diagnosed congenital anomalies- trend in tertiary care hospital. Int J Reprod Contracept Obstet Gynecol 2021;10:925-8. 